\title{
Systematic Discrepancies in Monte Carlo Predictions for Thin Surface Layers and Visualisation to Expose Root Causes
}

\author{
P. J .Statham* and P .Duncumb** \\ * Oxford Instruments Nanoanalysis, High Wycombe, Bucks HP12 3SE, U.K. \\ ** Willow House, Great Shelford, Cambridge, CB22 5LZ
}

Thickness and composition of thin layers on a substrate can be determined by measuring the "k-ratio", the ratio of intensity for a characteristic line to the intensity from a bulk pure specimen, for all emitted lines. Conversion from k-ratios to structure requires an accurate model for X-ray generation and this is commonly based on the assumption of a continuous phi-rho-z curve for each line. Useful weighting rules have been developed and successfully deployed to determine an "effective" phi-rho-z which works well for the analysis of many types of multilayer sample, although it is well recognised that this analytical model approach is questionable when there is a large atomic number difference between the various layers [1]. The obvious alternative is Monte Carlo (MC) simulation which can deal explicitly with sudden changes in material properties as individual electrons travel inside the specimen. The application of MC to stratified specimens is known to be useful in understanding how x-ray corrections behave for complex samples [2]. However, whereas analytical model approaches have converged on some programs that have been tested for practical analysis tasks, MC programs are often provided with many adjustable parameters and are usually intended as research tools rather than for routine analysis. With the widespread availability of some free MC programs and published examples showing excellent performance, there is an increasing expectation that these programs provide results that can replace real experiments. While there is no doubt these programs are useful, we would like to know if MC could ever match the accuracy of semi-empirical phi-rho-z programs for thin film analysis and deliver results well within $10 \%$ relative error. Therefore, we have investigated performance using the comprehensive data base of k-ratios measured on layered samples by Bastin \& Heijligers [3].

The first interesting aspect of this work was to decide which of a number of possible values for mass thickness to use. After careful analysis of the author's narrative [3], we decided that the RBS measured values provided the only unbiased measure of mass thickness, even though these were not the values tabulated. We also discovered a range of between 11.4 and $12.02 \mathrm{gm} / \mathrm{cm}^{3}$ quoted densities for Pd which emphasised the danger of making assumptions about density. We used versions of CASINO and NIST Monte that were downloaded from the web in July 2007. For CASINO, default simulation parameters were used, different scattering and cross section formulae were tried for NIST Monte and results from PENELOPE were calculated elsewhere [4]. We noted that conventional phi-rho-z analytical models predicted quite different results from the MCs, suggesting that there was no obvious reason to question the measured values. Some results are shown in fig. 1 where the ratio of MC-predicted and measured k-ratios is plotted against keV. PENELOPE is an extremely sophisticated simulation and as expected, shows less variation than the other programs. However, all the programs show systematic discrepancies sometimes exceeding $10 \%$ relative and this level of inaccuracy may not be acceptable in a routine analysis situation.. At first it appeared that all MC programs using CSDA showed a similar trend and the test with the discrete energy loss model within PENELOPE suggested that CSDA might explain some of the differences. Even PENELOPE showed a tendency to underestimate some cases so we tried to identify the root cause. 
To this end we wanted a method to visualise a large dataset of results that would expose all aspects of the data: film thickness, $\mathrm{keV}$, substrate and measured and predicted k-ratios. We also used a simple multiple scattering simulation to make rough estimates of the fraction of signal contributed by electrons backscattered from the substrate and wanted to include this in the display. Fig. 2 shows an example of the visualisation. The circles have sizes indicating 3 thicknesses of film and colours denoting the substrate. The sequence of circles of same colour and size left to right correspond to decreasing beam energies. The red circles below show the fraction generated by substrate bse and the same circle sizes allow this to be related to the results above. We have used this to unravel what are the sources of the systematic underestimate at low k-ratios and overestimate at high k-ratios.

\section{References}

[1] J. L. Pouchou, Microchim. Acta 138, 133-152 (2002)

[2] N. Ammann and P. Karduck, Microbeam Analysis-1990, 150-154 (1990)

[3] G. F. Bastin and H. J. M. Heijligers, X-Ray Spectrom., 29, 373-397 (2000)

[4] We are grateful to Xavier Llovet for running the PENELOPE simulations in November 2009 and Sam Denny for doing the simulations for NIST Monte and CASINO in August 2007

Fig.1. Calculated/Measured vs beam $\mathrm{keV}$ for different $\mathrm{MC}$ simulations

Fig.2. k-MC/k-measured for films with 11,45 and $181 \mathrm{ug} / \mathrm{cm}^{2} \mathrm{Pd}$ on different substrates. Estimated fraction of Pd L signal from substrate bse are circles in red.
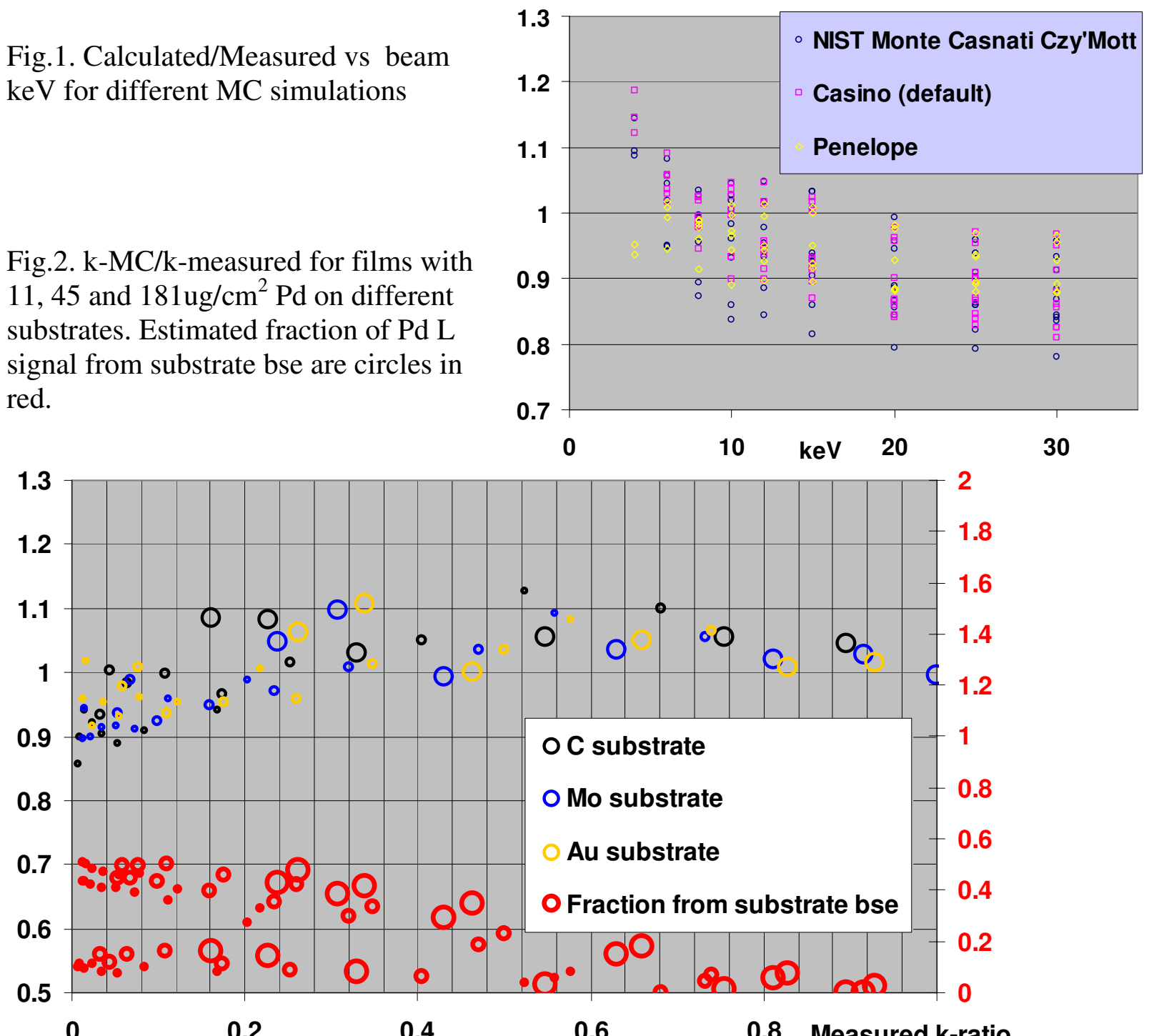